\title{
Differences in Breast Cancer Costs by Cancer Stage and Biomarker Subtype in New Zealand
}

\author{
Chunhuan Lao $^{1} \oplus \cdot$ Mohana Mondal $^{1} \cdot$ Marion Kuper-Hommel $^{2} \cdot$ lan Campbell ${ }^{3,4} \cdot$ Ross Lawrenson $^{1,5}$
}

Accepted: 26 January 2022 / Published online: 19 February 2022

(c) The Author(s) 2022

\begin{abstract}
Background Breast cancer requires the greatest expenditure among all cancer types, and the costs vary by cancer stage and biomarker status.

Objective This study aimed to examine the differences in public healthcare costs of breast cancer in New Zealand by stage and subtype.

Method This study included patients diagnosed with invasive breast cancer between 1 July 2010 and 30 June 2018 and receiving services in public hospitals. These patients were identified from the National Breast Cancer Register and/or New Zealand Cancer Registry. Linking with the Pharmaceutical Collection, National Minimum Dataset, National Non-Admitted Patient Collection, and Mortality Collection, we estimated the median public healthcare costs of breast cancer by cancer stage and biomarker subtype.

Results We identified 22,948 eligible patients. The median costs of breast cancer increased with stage of disease, from \$NZ26,930 for stage I disease to \$NZ50,388 for stage IV disease. The median costs for human epidermal growth factor receptor 2-positive (HER2+) disease were three times those for HER2-negative (HER2-) disease: \$NZ106,428 for HER2+ cancers compared with $\$ \mathrm{NZ28,481}$ for oestrogen receptor-positive (ER+)/HER2- cancers and $\$ N Z 31,722$ for triple negative disease. Over 55\% of the costs for HER2+ breast cancers were targeted therapy costs. For HER2- cancers, surgery incurred the biggest cost, followed by radiotherapy.

Conclusions Treating patients with early-stage breast cancer is less costly than treating those with metastatic disease. The costs vary considerably between the subtypes. Patients with HER2+ cancer incurred three times the costs of those with HER2 - cancers. These results provide baseline costing data for clinicians and policy makers when considering new targeted treatments.
\end{abstract}

Chunhuan Lao

chunhuan.lao@waikato.ac.nz

Mohana Mondal

mohana.mondal@waikato.ac.nz

Marion Kuper-Hommel

Marion.Kuper@waikatodhb.health.nz

Ian Campbell

Ian.campbell@waikatodhb.health.nz

Ross Lawrenson

Ross.Lawrenson@waikatodhb.health.nz
1 Medical Research Centre, Te Huataki Waiora - School of Health, The University of Waikato, Private Bag 3105, Hamilton 3240, New Zealand

2 Medical Oncology, Waikato District Health Board, Hamilton, New Zealand

3 School of Medicine, The University of Auckland, Auckland, New Zealand

4 General Surgery, Waikato District Health Board, Hamilton, New Zealand

5 Strategy and Funding, Waikato District Health Board, Hamilton, New Zealand 


\section{Key Points for Decision Makers}

Treating patients with early-stage breast cancer is less costly than treating those with metastatic disease.

Patients with human epidermal growth factor receptor 2-positive (HER2+) cancer had three times the costs of those with HER2- cancers.

\section{Introduction}

Breast cancer is the most common cancer in New Zealand women [1]. Every year, around 3000 new cases are diagnosed in New Zealand, and around 600 deaths are specific to breast cancer [2]. Breast cancer is also one of the cancers that requires the greatest expenditure [3-5]. A New Zealand study by Blakely et al. [5] showed that breast cancer was the second-most expensive cancer (following colorectal cancer), costing New Zealand dollars (\$NZ)126.7 million per year and accounting for $14 \%$ of total cancer costs [5].

Breast cancer stage and biomarker status (oestrogen receptor [ER], progesterone receptor [PR], and human epidermal growth factor receptor 2 positivity $[$ HER2+]) are important predictive and prognostic indicators for breast cancer and affect treatment decision making [6, 7]. Patients diagnosed with stage I breast cancer are more likely to have breast-conserving surgery than mastectomy compared with those with stage II or III breast cancer [8]. Patients with metastatic breast cancer are most likely to have systemic treatments as the main treatment [9]. Surgery remains a mainstay of treatment for stage I-III breast cancer; however, adjuvant systemic treatments, including targeted therapy, and adjuvant radiation therapy lower the risk of recurrence and have been responsible for major improvements in survival over the last 40 years $[10,11]$. For patients with hormone receptor-positive (ER+ and/or PR+) breast cancer, tamoxifen or aromatase inhibitors are usually recommended for at least 5 years [12]. HER2 targeted treatments, including trastuzumab, have been reported to be beneficial for improving the survival of patients with HER2+ disease $[11,13]$.

Breast cancer costs also vary by cancer stage and biomarker status. A recent systematic review showed that the mean costs of breast cancer at stage II, III, and IV were 32, 95 , and $109 \%$ higher than at stage I, and the mean costs of regional and distant breast cancer were 41 and $165 \%$ higher than for local breast cancer [6]. Some systemic treatments that are specific to biomarker subtypes are expensive, which results in great variations in treatment costs by biomarker subtype [11]. We conducted this study to examine the differences in public healthcare costs of breast cancer in New Zealand by stage and subtype.

\section{Methods}

\subsection{Data Sources}

Patients diagnosed with invasive (stage I-IV) breast cancer between 1 July 2010 and 30 June 2018 were identified from the National Breast Cancer Register and the New Zealand Cancer Registry (NZCR). We excluded patients who only received treatments for breast cancer in private hospitals and included those who received healthcare services in public hospitals (with or without private treatments). Eligible patients were linked with the Pharmaceutical Collection (PHARMS, including all publicly funded pharmaceuticals prescribed in both public and private hospitals), National Minimum Dataset (NMDS, including all publicly funded inpatient records), National Non-Admitted Patients Collection (NNAPC, including all publicly funded outpatient records), and the Mortality Collection (MORT, coded mortality information), datasets and death certificates (uncoded mortality information) using the national health index number, which is a unique identifier for people using publicly funded health and disability services in New Zealand.

Based on their prognosis and treatment pattern, breast cancer subtypes were categorised into three groups according to biomarker status: ER+/HER2-, HER2+, and triple negative. In this study, HER2+ was defined as fluorescence in situ hybridization-amplified or 3+ staining on immunohistochemistry according to the 2013 American Society of Clinical Oncology Guideline [14]. As recommended in the 2001 St. Gallen Consensus, ER+ or PR+ was assessed as any degree of immunohistochemistry positivity (at least $1+$ intensity and $1 \%$ staining of nuclei) [15].

\subsection{Cancer Care Pathway}

We divided the cancer care pathway into two phases: (1) the initial treatment phase (TP, 3 months preceding and 12 months following diagnosis of breast cancer) and (2) the follow-up phase (second to fifth year following diagnosis). We further broke down the follow-up phase into the second (FU2), third (FU3), fourth (FU4), and fifth (FU5) year. The 5 -year follow-up time was because eligible patients were often recommended for 5-10 years of endocrine therapy. We considered the earliest of date of death or the latest date of service (31 December 2019) available in the NNAPC, NMDS, and PHARMS as the censor date. The estimation of costs for each phase only included patients who had follow-up time for that phase. To calculate the total cost of all 
phases combined, we only included patients who had followup time for all phases.

\subsection{Cost Estimation}

The cost estimation was from the perspective of the New Zealand Ministry of Health and only included public medical costs: public outpatient services, public inpatient services, and funded pharmaceuticals (prescribed by either public or private hospitals). Our clinical advisers (RL, MKH, and IC) checked the definitions of purchase unit codes for outpatient services (in NNAPC), the definitions of surgery codes for inpatient services (in NMDS), and pharmaceuticals, and this study only included the inpatient, outpatient, and pharmaceutical records relevant to breast cancer. Costs of diagnostic services such as radiology and pathology and of treatment response assessment tools were also included in the inpatient or outpatient costs. For pharmaceuticals, only relevant endocrine therapy, chemotherapy, and HER2-targeted therapies were included. Other medications such as pain killers were not included, because we could not identify whether these medications were breast cancer related. Including all these medications would overestimate the costs. All cost estimations were based on $\$ N Z$, year 2019/2020 values. In terms of the purchasing power, $\$ N Z 1$ was equal to $£ 0.484$ and \$US0.692 in 2020. Appendixes 1-3 in the electronic supplementary material include the lists of included outpatient events, inpatient events, and pharmaceuticals, respectively.

Outpatient costs were estimated by multiplying the number of relevant outpatient visits recorded in the NNAPC with the unit cost of outpatient visits. The unit costs for outpatient visits were based on district health board-contracted purchase unit prices [16]. Inpatient costs were estimated by multiplying the accumulated cost weights for all relevant events by the purchase unit price as set by the national pricing programme [16]. The Ministry of Health calculates the cost weights, which provide resource utilisation information, for each diagnosis-related group code using the weighted inlier equivalent separation method, and sets a purchase unit price for each year. The 2019/2020 cost-weight unit price was \$NZ5216.21 [17]. The costs of publicly funded pharmaceuticals were estimated by multiplying the quantity of the pharmaceuticals dispensed by the unit prices for each pharmaceutical that appears in the pharmaceutical schedule [18].

Because costs are right-skewed data, we estimated the median and interquartile range (IQR) of the public medical costs of breast cancer during the initial TP and the follow-up phase. The costs were also computed by tumour anatomic stage (stage I, II, III, and IV) [19] and by biomarker subtype. The difference in median costs by tumour anatomic stage and by biomarker subtype were examined using the median test, and the subgroup difference was considered significant if the $p$ value was $<0.05$. We also calculated the proportion of different cost components in total costs: surgery costs; costs of diagnostic tests, scans, or biopsies; radiotherapy costs; costs of medical oncology visits; chemotherapy costs; targeted therapy cost; endocrine therapy costs; and other costs.

\section{Results}

Between 1 July 2010 and 30 June 2018, a total of 22,948 patients were diagnosed with invasive breast cancer and received treatments for breast cancer in public hospitals (Table 1), including 6405 patients with stage I cancers, 6744 with stage II, 3828 with stage III, 1093 with stage IV, and 4878 with an unknown cancer stage. The majority of the cancer cases were ER+/HER2- (15,615 [74\%]); 3384 (16\%) were HER2+ and 1963 (9\%) were triple negative. The proportion of ER+/HER2 disease decreased with increasing cancer stage, from $80 \%$ for stage I cancers to $62 \%$ for stage IV cancers. In contrast, the percentage of HER2+ cancers increased with cancer stage, from $7 \%$ for stage I disease to $27 \%$ for stage IV. The number of eligible patients in each phase decreased with time because some patients died or were censored because of loss of follow-up.

The median costs of breast cancer for the whole study period (TP-FU5) were \$NZ26,930 (IQR 12,479-41,512) for stage I disease, \$NZ31,372 (IQR 18,563-50,933) for stage II disease, \$NZ42,273 (IQR 25,681-75,162) for stage III disease, and \$NZ50,388 (IQR 20,685-116,161) for stage IV disease (median test $p<0.001$; Table 2). Stage III cancers had the highest costs during the TP, and stage IV cancers had the lowest costs during the TP. The costs in FU2-FU5 for stage IV cancers were consistently higher than the costs for other cancer stages.

The median costs for HER2+ disease were three times those for HER2 - disease (Table 2): \$NZ106,428 (IQR 54,350-139,103) for HER2+ cancers compared with \$NZ28,481 (IQR 17,333-42,601) for ER+/HER2- cancers and \$NZ31,722 (IQR 17,714-47,283) for triple negative disease $(p<0.001)$. HER2+ cancers had higher costs during all phases than HER2- cancers. For cancers of the same subtype, the costs increased with cancer stage. For example, the median costs increased from $\$$ NZ25,485 (IQR 11,257-37,248) for ER+/HER2- stage I cancers to $\$ N Z 38,855$ (IQR $18,826-61,429$ ) for stage IV cancers $(p<0.001)$, and the median costs increased from \$NZ74,290 $(27,117-115,479)$ for HER2+ stage I cancers to \$NZ279,644 $(188,778-381,531)$ for HER2+ stage IV cancers $(p<0.001)$.

Generally, the proportions of total costs accounted for by surgery and diagnostic tests, scans, or biopsies decreased with increasing cancer stage in all phases (Table 3). During the TP, the proportion of costs applicable to surgery ranged 
Table 1 Number of eligible patients by cancer stage and by subtype

\begin{tabular}{|c|c|c|c|c|c|c|}
\hline Subgroup & $\mathrm{TP}, n(\%)$ & FU2 & FU3 & FU4 & FU5 & All years \\
\hline Overall & 22,948 & 22,204 & 19,918 & 16,527 & 13,312 & 13,312 \\
\hline \multicolumn{7}{|l|}{ By stage } \\
\hline Stage I & $6405(35)$ & 6373 & 5733 & 4564 & 3539 & 3539 \\
\hline Stage II & $6744(37)$ & 6676 & 6101 & 5129 & 4200 & 4200 \\
\hline Stage III & $3828(21)$ & 3737 & 3377 & 2845 & 2365 & 2365 \\
\hline Stage IV & $1093(6)$ & 753 & 524 & 359 & 254 & 254 \\
\hline Unknown & 4878 & 4665 & 4183 & 3630 & 2954 & 2954 \\
\hline \multicolumn{7}{|l|}{ By subtype } \\
\hline ER+/HER2- & $15,615(74)$ & 15,320 & 13,911 & 11,652 & 9438 & 9438 \\
\hline HER2+ & $3384(16)$ & 3289 & 2954 & 2427 & 1907 & 1907 \\
\hline Triple negative & $1963(9)$ & 1852 & 1571 & 1263 & 996 & 996 \\
\hline Unknown & 1986 & 1743 & 1482 & 1185 & 971 & 971 \\
\hline \multicolumn{7}{|l|}{ Stage I } \\
\hline ER+/HER2- & $4796(80)$ & 4775 & 4304 & 3417 & 2643 & 2643 \\
\hline HER2+ & 787 (13) & 784 & 697 & 560 & 418 & 418 \\
\hline Triple negative & $428(7)$ & 422 & 372 & 282 & 223 & 223 \\
\hline Unknown & 394 & 392 & 360 & 305 & 255 & 255 \\
\hline \multicolumn{7}{|l|}{ Stage II } \\
\hline ER+/HER2- & $4807(75)$ & 4768 & 4379 & 3720 & 3075 & 3075 \\
\hline HER 2+ & $1012(16)$ & 1003 & 918 & 754 & 593 & 593 \\
\hline Triple negative & $610(9)$ & 598 & 532 & 439 & 347 & 347 \\
\hline Unknown & 315 & 307 & 272 & 216 & 185 & 185 \\
\hline \multicolumn{7}{|l|}{ Stage III } \\
\hline ER+/HER2- & $2452(67)$ & 2412 & 2191 & 1878 & 1562 & 1562 \\
\hline HER 2+ & $777(21)$ & 763 & 708 & 603 & 507 & 507 \\
\hline Triple negative & $444(12)$ & 424 & 363 & 284 & 225 & 225 \\
\hline Unknown & 155 & 138 & 115 & 80 & 71 & 71 \\
\hline \multicolumn{7}{|l|}{ Stage IV } \\
\hline ER+/HER2- & $511(62)$ & 395 & 286 & 202 & 138 & 138 \\
\hline HER2+ & $218(27)$ & 169 & 117 & 77 & 53 & 53 \\
\hline Triple negative & $91(11)$ & 44 & 21 & 17 & 13 & 13 \\
\hline Unknown & 273 & 145 & 100 & 63 & 50 & 50 \\
\hline
\end{tabular}

ER oestrogen receptor, FU2 second follow-up year, FU3 third follow-up year, FU4 fourth follow-up year, FU5 fifth follow-up year, HER2+/- human epidermal growth factor receptor 2 positive/negative, TP initial treatment phase from $37 \%$ for stage I cancers to $26 \%$ for stage IV cancers, and the proportion of the costs accounted for by diagnostic tests, scans, or biopsies ranged from 21 to $12 \%$. Conversely, the percentage of total costs incurred by medical oncology visits, targeted therapy costs, and chemotherapy costs increased with cancer stage. During the TP, the percentage of total costs from medical oncology visits ranged from 3\% for stage I cancers to $7 \%$ for stage IV cancers, the percentage for targeted therapy costs ranged from 14 to $31 \%$, and the percentage for chemotherapy ranged from 4 to $9 \%$.

For HER2+ cancers, targeted therapy costs accounted for more than half of the total costs during the TP and $72 \%$ during the FU2 (Table 4). For ER+/HER2- cancers, surgery incurred the highest costs (44\% during the TP) followed by radiotherapy ( $22 \%$ during the TP). Chemotherapy costs only accounted for 5\% of the total costs for ER+/HER2- disease during the TP and $11 \%$ for triple negative cancers.

\section{Discussion}

The main findings from this study were that the costs of breast cancer varied substantially by stage and subtype. The median costs of stage II, III, and IV cancers were 16,57 , and $87 \%$ higher than the costs of stage I cancers, and the costs of HER $2+$ disease were more than three times the costs of the HER2- cancers. These are consistent with the results from previous studies that have also demonstrated a higher cost with increasing stage and/or a higher cost for HER2+ tumours than HER2- disease [6, 20, 21]. The results from 
Table 2 Median costs $(\$ N Z)$ and interquartile range

\begin{tabular}{|c|c|c|c|c|c|c|}
\hline Stage & $\mathrm{TP}$ & FU2 & FU3 & FU4 & FU5 & All years \\
\hline Overall & $\begin{array}{l}25,855(12,401- \\
39,719)\end{array}$ & $483(43-2529)$ & $161(15-856)$ & $94(0-595)$ & $64(0-542)$ & $\begin{array}{l}30,823(17,952- \\
51,823)\end{array}$ \\
\hline \multicolumn{7}{|l|}{ By stage } \\
\hline Stage I & $\begin{array}{l}22,588(10,098- \\
34,027)\end{array}$ & 393 (30-959) & $64(0-495)$ & $61(0-436)$ & $56(0-408)$ & $\begin{array}{l}26,930(12,479- \\
\quad 41,512)\end{array}$ \\
\hline Stage II & $\begin{array}{l}26,504(15,242- \\
\quad 40,242)\end{array}$ & $505(43-1915)$ & $189(14-814)$ & $95(10-580)$ & $64(0-537)$ & $\begin{array}{l}31,372(18,563- \\
50,933)\end{array}$ \\
\hline Stage III & $\begin{array}{l}32,226(19,016- \\
\quad 49,771)\end{array}$ & 1074 (393-9416) & $542(56-1547)$ & $457(42-1104)$ & 407 (28-922) & $\begin{array}{l}42,273(25,681- \\
\quad 75,162)\end{array}$ \\
\hline Stage IV & $\begin{array}{l}17,737(4,294- \\
43,291)\end{array}$ & $\begin{array}{l}5617(1213- \\
21,864)\end{array}$ & $3835(899-19,897)$ & $3561(565-16,996)$ & $3070(462-13,410)$ & $\begin{array}{l}50,388(20,685- \\
116,161)\end{array}$ \\
\hline$p$ value & $<0.001$ & $<0.001$ & $<0.001$ & $<0.001$ & $<0.001$ & $<0.001$ \\
\hline \multicolumn{7}{|l|}{ By subtype } \\
\hline ER+/HER2- & $\begin{array}{l}23,467(11,560- \\
34,777)\end{array}$ & $437(40-1146)$ & $149(30-800)$ & $95(16-561)$ & $64(11-528)$ & $\begin{array}{l}28,481(17,333- \\
\quad 42,601)\end{array}$ \\
\hline HER2+ & $\begin{array}{l}\text { 71,081 (29,273- } \\
93,070)\end{array}$ & $\begin{array}{l}20,261(1218- \\
34,411)\end{array}$ & $457(30-1554)$ & $393(14-1049)$ & $126(0-898)$ & $\begin{array}{l}106,428(54,350- \\
139,103)\end{array}$ \\
\hline Triple negative & $\begin{array}{l}26,275(14,050- \\
\quad 37,461)\end{array}$ & $393(0-1843)$ & $64(0-800)$ & $3(0-457)$ & $0(0-407)$ & $\begin{array}{l}31,722(17,714- \\
47,283)\end{array}$ \\
\hline$p$ value & $<0.001$ & $<0.001$ & $<0.001$ & $<0.001$ & $<0.001$ & $<0.001$ \\
\hline \multicolumn{7}{|l|}{ Stage I } \\
\hline ER+/HER2- & $\begin{array}{l}20,826(9778- \\
30,204)\end{array}$ & $254(30-809)$ & $64(0-467)$ & $60(0-423)$ & $56(0-407)$ & $\begin{array}{l}25,485(11,257- \\
\quad 37,248)\end{array}$ \\
\hline HER2+ & $\begin{array}{l}54,704(19,629- \\
85,053)\end{array}$ & $\begin{array}{c}11,816(393- \\
25,463)\end{array}$ & $125(0-722)$ & $74(0-551)$ & $60(0-546)$ & $\begin{array}{l}74,290(27,117- \\
115,479)\end{array}$ \\
\hline Triple negative & $\begin{array}{l}22,908(8343- \\
34,614)\end{array}$ & $129(0-814)$ & $0(0-457)$ & $64(0-413)$ & $0(0-407)$ & $\begin{array}{l}25,581(8296- \\
42,950)\end{array}$ \\
\hline$p$ value & $<0.001$ & $<0.001$ & $<0.001$ & 0.059 & 0.360 & $<0.001$ \\
\hline \multicolumn{7}{|l|}{ Stage II } \\
\hline $\mathrm{ER}+/ \mathrm{HER} 2-$ & $\begin{array}{l}24,341(13,128- \\
35,396)\end{array}$ & $449(40-1065)$ & $174(20-707)$ & $104(23-569)$ & $64(10-533)$ & $\begin{array}{l}28,621(17,647- \\
42,261)\end{array}$ \\
\hline HER2+ & $\begin{array}{l}69,679(29,004- \\
91,176)\end{array}$ & $\begin{array}{l}20,684(1064- \\
33,333)\end{array}$ & 467 (29-1316) & $393(0-890)$ & $104(0-610)$ & $\begin{array}{l}101,870(51,568- \\
131,688)\end{array}$ \\
\hline Triple negative & $\begin{array}{l}26,032(14,552- \\
37,220)\end{array}$ & $407(0-1501)$ & $24(0-786)$ & $0(0-407)$ & $0(0-407)$ & $\begin{array}{l}32,525(17,926- \\
\quad 47,269)\end{array}$ \\
\hline$p$ value & $<0.001$ & $<0.001$ & $<0.001$ & $<0.001$ & $<0.001$ & $<0.001$ \\
\hline \multicolumn{7}{|l|}{ Stage III } \\
\hline ER+/HER2- & $\begin{array}{l}28,662(18,061- \\
\quad 40,816)\end{array}$ & $990(407-2492)$ & $547(64-1381)$ & $505(56-1094)$ & 433 (42-946) & $\begin{array}{l}35,748(22,810- \\
53,275)\end{array}$ \\
\hline HER2+ & $\begin{array}{l}77,510(43,501- \\
95,584)\end{array}$ & $\begin{array}{l}26,858(8416- \\
40,991)\end{array}$ & $554(56-2132)$ & $505(40-1513)$ & $407(10-1051)$ & $\begin{array}{l}118,172(80,016- \\
150,747)\end{array}$ \\
\hline Triple negative & $\begin{array}{l}29,488(17,723- \\
39,716)\end{array}$ & $505(0-2951)$ & $393(0-1860)$ & $20(0-545)$ & $0(0-449)$ & $\begin{array}{l}34,628(22,628- \\
\quad 49,827)\end{array}$ \\
\hline$p$ value & $<0.001$ & $<0.001$ & $<0.001$ & $<0.001$ & $<0.001$ & $<0.001$ \\
\hline \multicolumn{7}{|l|}{ Stage IV } \\
\hline ER+/HER2- & $\begin{array}{l}14,607(4874- \\
29,520)\end{array}$ & $\begin{array}{c}3072(1065- \\
10,717)\end{array}$ & 2767 (534-9246) & $2528(467-9230)$ & 2697 (498-8723) & $\begin{array}{l}38,855(18,826- \\
\quad 61,429)\end{array}$ \\
\hline HER2+ & $\begin{array}{l}80,738(30,499- \\
106,337)\end{array}$ & $\begin{array}{l}39,593(11,625- \\
82,200)\end{array}$ & $\begin{array}{l}35,035(3589- \\
82,168)\end{array}$ & $\begin{array}{l}28,028(6594- \\
76,709)\end{array}$ & $\begin{array}{l}23,123(1815- \\
67,262)\end{array}$ & $\begin{array}{l}279,644(188,778- \\
381,531)\end{array}$ \\
\hline Triple negative & $\begin{array}{l}29,168(12,757- \\
\quad 43,940)\end{array}$ & $\begin{array}{l}7860(1133- \\
23,349)\end{array}$ & $\begin{array}{c}5769(1009- \\
13,033)\end{array}$ & $1300(229-6633)$ & $2737(449-19,144)$ & $\begin{array}{l}65,380(31,744- \\
89,291)\end{array}$ \\
\hline$p$ value & $<0.001$ & $<0.001$ & $<0.001$ & $<0.001$ & $<0.001$ & $<0.001$ \\
\hline
\end{tabular}

ER oestrogen receptor, FU2 second follow-up year, FU3 third follow-up year, FU4 fourth follow-up year, FU5 fifth follow-up year, HER2+/human epidermal growth factor receptor 2 positive/negative, $T P$ initial treatment phase 
Table 3 Proportion (\%) of each cost component in different phases by cancer stage

\begin{tabular}{|c|c|c|c|c|c|}
\hline Cancer stage & Stage I & Stage II & Stage III & Stage IV & Overall \\
\hline \multicolumn{6}{|l|}{$\mathrm{TP}$} \\
\hline Surgery & 37 & 37 & 33 & 26 & 35 \\
\hline Diagnostic test, scan or biopsy & 21 & 18 & 11 & 12 & 16 \\
\hline Radiotherapy & 18 & 16 & 17 & 12 & 17 \\
\hline Medical oncology & 3 & 4 & 7 & 7 & 4 \\
\hline Targeted therapy & 14 & 16 & 20 & 31 & 18 \\
\hline Chemotherapy & 4 & 6 & 10 & 9 & 7 \\
\hline Endocrine therapy & 0 & 0 & 0 & 0 & 0 \\
\hline Others & 3 & 3 & 2 & 2 & 2 \\
\hline \multicolumn{6}{|l|}{ FU2 } \\
\hline Surgery & 26 & 24 & 21 & 17 & 22 \\
\hline Diagnostic test, scan or biopsy & 10 & 7 & 5 & 6 & 7 \\
\hline Radiotherapy & 5 & 5 & 6 & 8 & 6 \\
\hline Medical oncology & 6 & 7 & 8 & 11 & 8 \\
\hline Targeted therapy & 42 & 47 & 49 & 47 & 47 \\
\hline Chemotherapy & 7 & 7 & 8 & 10 & 8 \\
\hline Endocrine therapy & 1 & 1 & 0 & 0 & 0 \\
\hline Others & 5 & 3 & 2 & 1 & 3 \\
\hline \multicolumn{6}{|l|}{ FU3 } \\
\hline Surgery & 48 & 43 & 44 & 16 & 39 \\
\hline Diagnostic test, scan or biopsy & 15 & 14 & 8 & 7 & 11 \\
\hline Radiotherapy & 11 & 8 & 7 & 9 & 9 \\
\hline Medical oncology & 7 & 10 & 12 & 11 & 10 \\
\hline Targeted therapy & 7 & 13 & 18 & 45 & 20 \\
\hline Chemotherapy & 4 & 5 & 6 & 11 & 6 \\
\hline Endocrine therapy & 1 & 1 & 1 & 0 & 1 \\
\hline Others & 7 & 5 & 3 & 1 & 4 \\
\hline \multicolumn{6}{|l|}{ FU4 } \\
\hline Surgery & 50 & 39 & 40 & 16 & 38 \\
\hline Diagnostic test, scan or biopsy & 17 & 15 & 8 & 6 & 11 \\
\hline Radiotherapy & 9 & 8 & 7 & 6 & 8 \\
\hline Medical oncology & 6 & 10 & 11 & 13 & 10 \\
\hline Targeted therapy & 7 & 16 & 24 & 44 & 22 \\
\hline Chemotherapy & 3 & 6 & 7 & 13 & 7 \\
\hline Endocrine therapy & 1 & 1 & 1 & 0 & 1 \\
\hline Others & 6 & 5 & 2 & 2 & 3 \\
\hline \multicolumn{6}{|l|}{ FU5 } \\
\hline Surgery & 39 & 36 & 37 & 18 & 34 \\
\hline Diagnostic test, scan or biopsy & 17 & 14 & 6 & 3 & 10 \\
\hline Radiotherapy & 12 & 9 & 9 & 8 & 9 \\
\hline Medical oncology & 6 & 10 & 12 & 13 & 10 \\
\hline Targeted therapy & 15 & 20 & 25 & 43 & 25 \\
\hline Chemotherapy & 5 & 6 & 7 & 13 & 8 \\
\hline Endocrine therapy & 1 & 1 & 1 & 0 & 1 \\
\hline Others & 4 & 4 & 3 & 2 & 3 \\
\hline
\end{tabular}

ER oestrogen receptor, FU2 second follow-up year, FU3 third follow-up year, FU4 fourth follow-up year, FU5 fifth follow-up year, HER2+/- human epidermal growth factor receptor 2 positive/negative, TP initial treatment phase 
Table 4 Proportion (\%) of each cost component in different phases by cancer subtype

\begin{tabular}{|c|c|c|c|c|}
\hline Cancer subtype & ER+/HER2- & HER2+ & Triple negative & Overall \\
\hline \multicolumn{5}{|l|}{ TP } \\
\hline Surgery & 44 & 18 & 39 & 35 \\
\hline Diagnostic test, scan or biopsy & 22 & 7 & 17 & 16 \\
\hline Radiotherapy & 21 & 9 & 21 & 17 \\
\hline Medical oncology & 4 & 5 & 7 & 4 \\
\hline Targeted therapy & 1 & 51 & 1 & 18 \\
\hline Chemotherapy & 5 & 10 & 11 & 7 \\
\hline Endocrine therapy & 0 & 0 & 0 & 0 \\
\hline Others & 3 & 2 & 3 & 2 \\
\hline \multicolumn{5}{|l|}{ FU2 } \\
\hline Surgery & 46 & 8 & 44 & 22 \\
\hline Diagnostic test, scan or biopsy & 14 & 3 & 13 & 7 \\
\hline Radiotherapy & 11 & 2 & 15 & 6 \\
\hline Medical oncology & 12 & 5 & 10 & 8 \\
\hline Targeted therapy & 7 & 72 & 7 & 47 \\
\hline Chemotherapy & 5 & 9 & 5 & 8 \\
\hline Endocrine therapy & 1 & 0 & 0 & 0 \\
\hline Others & 5 & 1 & 5 & 3 \\
\hline \multicolumn{5}{|l|}{ FU3 } \\
\hline Surgery & 50 & 22 & 46 & 39 \\
\hline Diagnostic test, scan or biopsy & 14 & 6 & 13 & 11 \\
\hline Radiotherapy & 10 & 5 & 11 & 9 \\
\hline Medical oncology & 11 & 8 & 11 & 10 \\
\hline Targeted therapy & 3 & 48 & 7 & 20 \\
\hline Chemotherapy & 5 & 8 & 8 & 6 \\
\hline Endocrine therapy & 1 & 0 & 0 & 1 \\
\hline Others & 5 & 2 & 4 & 4 \\
\hline \multicolumn{5}{|l|}{ FU4 } \\
\hline Surgery & 47 & 22 & 53 & 38 \\
\hline Diagnostic test, scan or biopsy & 15 & 5 & 15 & 11 \\
\hline Radiotherapy & 10 & 3 & 9 & 8 \\
\hline Medical oncology & 12 & 8 & 9 & 10 \\
\hline Targeted therapy & 3 & 52 & 5 & 22 \\
\hline Chemotherapy & 6 & 8 & 5 & 7 \\
\hline Endocrine therapy & 2 & 0 & 0 & 1 \\
\hline Others & 5 & 2 & 3 & 3 \\
\hline \multicolumn{5}{|l|}{ FU5 } \\
\hline Surgery & 42 & 15 & 53 & 34 \\
\hline Diagnostic test, scan or biopsy & 15 & 2 & 15 & 10 \\
\hline Radiotherapy & 12 & 4 & 10 & 9 \\
\hline Medical oncology & 13 & 8 & 7 & 10 \\
\hline Targeted therapy & 5 & 61 & 6 & 25 \\
\hline Chemotherapy & 8 & 8 & 5 & 8 \\
\hline Endocrine therapy & 2 & 0 & 0 & 1 \\
\hline Others & 4 & 2 & 3 & 3 \\
\hline
\end{tabular}

ER oestrogen receptor, FU2 second follow-up year, FU3 third follow-up year, FU4 fourth follow-up year, FU5 fifth follow-up year, HER2+/- human epidermal growth factor receptor 2 positive/negative, $T P$ initial treatment phase 
this study are important for healthcare planning, including decisions around medical resource distribution, as well as budget impact analysis for funding a new medication that is targeted for a subtype.

The differences in costs by cancer stage and subtype can be attributed to the differences in treatments and risk of cancer progression of different subgroups. For example, patients with metastatic breast cancer are less likely to receive surgical treatments than those with other cancer stages, which explains the lower surgery costs during the TP. The mainstay of treatments for stage IV cancers-systemic treatmentsare included in the pharmaceutical costs [22]. Patients with stage II and III breast cancers are at higher risk of developing metastatic disease than are those with stage I cancers [23] and are therefore more likely to incur healthcare costs in subsequent years. Breast cancer screening also affects the distribution of cancer subtype at diagnosis. Screeningdetected patients are more likely to have ER+/HER2- cancers [24]. These would result in lower mean costs per case for screen-detected cancers. However, screening is also associated with overdiagnosis and overtreatment and thus would increase total costs [25].

HER2+ cancers incurred higher costs primarily because of expensive targeted therapy, mainly HER2-targeted therapies. Over half of the costs for HER2+ disease were targeted therapy costs. During the study period of 1 July 2010 and 31 December 2019, trastuzumab, pertuzumab, and ado-trastuzumab emtansine (DM-1) were funded by PHARMAC to treat HER2+ breast cancer in New Zealand. Trastuzumab has been funded for HER2+ metastatic breast cancer since 2002 and for HER2+ stage I-III breast cancer since 2007 [26].

Some treatments, such as endocrine therapy (e.g. tamoxifen, aromatase inhibitors) are taken for a long time but have relatively low costs. Other targeted treatments, such as HER2-targeted therapies, have been shown to improve survival [11] but are expensive. The cost of a 12-month sequential treatment of trastuzumab continuing after initial taxanebased concurrent therapy with trastuzumab is \$NZ71,000 per patient per annum [27]. With the increasing incidence of breast cancer, the economic burden of targeted treatments is expected to increase [28]. To control the increasing costs, the funder would either need to have a better price for targeted treatments or to move to generic drugs after the patent expires.

The cost of breast cancer is a changing landscape, with new treatments becoming available and funded in New Zealand. Pertuzumab (funded since January 2017) and adotrastuzumab emtansine (since 1 December 2019) were only recently approved by PHARMAC for HER2+ advanced breast cancer [29]. Therefore, the costs of these two medications did not account for a large proportion of the total costs in our study but may further increase the costs of treating these patients in the future. Palbociclib, a targeting cyclindependent kinase 4/6 inhibitor, has been funded in New Zealand since April 2020. Some biosimilars for targeted therapies are now available for breast cancer, which may reduce future treatment costs [30]. Further studies will be needed to estimate the impact of newly funded medications on the costs of breast cancer.

One of the strengths of this study is that we combined the National Breast Cancer Register data with the NZCR, PHARMS, NMDS, NNAPC, and MORT datasets, and death certificates, so we had comprehensive data on all patients with breast cancer. This provided a large cohort of patients with detailed information on cancer stage and subtype. One limitation of this study was that, to calculate the pharmaceutical costs, we used the unit costs of drugs available in the pharmaceutical schedule. Using these unit costs might lead to an overestimation of the costs of cancer, because we did not know whether any confidential rebates existed for these drugs [31]. Over $20 \%$ of patients had cancer of an unknown stage. Most were identified from the NZCR, which mainly applies the Surveillance Epidemiology and End Results programme cancer staging definitions. While we reported the costs each year following diagnosis, we censored patients who died or reached their censor date, which means the number of patients reduced in the later years (especially in FU4 and FU5). Another limitation is that we included only endocrine therapy, chemotherapy, and HER2-targeted therapy but no other drugs that might have been used for breast cancer, e.g. anti-emetic drugs, growth factors, pain killers, and bisphosphonates, because we could not identify whether these drugs were used for breast cancer or other diseases, e.g. arthritis and osteoporosis. This study only estimated public healthcare costs. More research is needed on private and patient costs. Access to systemic treatments in New Zealand is more limited than in other developed countries. The cost differences by tumour anatomic stage and by biomarker subtype may be greater in countries with more liberal access to new targeted therapies.

\section{Conclusions}

Treating patients with early-stage breast cancer is less costly than treating those with metastatic disease. The costs vary considerably between different subtypes. Patients with HER 2+ cancer had three times the costs of those with HER2 - disease. These results provide baseline costing data for clinicians and policy makers considering new targeted treatments.

Supplementary Information The online version contains supplementary material available at https://doi.org/10.1007/s41669-022-00327-5. 
Acknowledgements The authors acknowledge the Breast Cancer Foundation New Zealand and the Health Research Council of New Zealand for the financial support and the National Breast Cancer Register and the Ministry of Health for providing the detailed data.

\section{Declarations}

Funding This study was funded by the Health Research Council of New Zealand and Breast Cancer Foundation New Zealand.

Conflict of interest Chunhuan Lao, Mohana Mondal, Marion KuperHommel, Ian Campbell, and Ross Lawrenson have no conflicts of interest that are directly relevant to the content of this article.

Availability of data and material The data used for this study are not publicly available because of the ethics for patient information. They can be accessed through the National Breast Cancer Register and the Ministry of Health with appropriate ethics approval.

Code availability Not applicable.

Author contributions All authors contributed to the study conception and design. Material was prepared and data acquired by CL. Data were cleaned and analysed by MM and CL. The first draft of the manuscript was written by CL, MM, and RL, and all authors commented on subsequent versions of the manuscript. All authors read and approved the final manuscript.

Ethics approval Ethics approval for the study was granted through the Northern B Health and Disability Ethics Committee, reference: 19/ NTB/188.

Consent Not applicable.

Open Access This article is licensed under a Creative Commons Attribution-NonCommercial 4.0 International License, which permits any non-commercial use, sharing, adaptation, distribution and reproduction in any medium or format, as long as you give appropriate credit to the original author(s) and the source, provide a link to the Creative Commons licence, and indicate if changes were made. The images or other third party material in this article are included in the article's Creative Commons licence, unless indicated otherwise in a credit line to the material. If material is not included in the article's Creative Commons licence and your intended use is not permitted by statutory regulation or exceeds the permitted use, you will need to obtain permission directly from the copyright holder. To view a copy of this licence, visit http://creativecommons.org/licenses/by-nc/4.0/.

\section{References}

1. Ministry of Health. Breast cancer. 2018. https://www.health.govt. nz/your-health/conditions-and-treatments/diseases-and-illnesses/ breast-cancer. Cited 20 Feb 2019.

2. Ministry of Health. New cancer registrations 2015. 2017. https:// www.health.govt.nz/publication/new-cancer-registrations-2015. Cited 20 Feb 2019.

3. Ministry of Health. The price of cancer: the public price of registered cancer in New Zealand. 2011: Wellington: Ministry of Health.

4. Lao C, et al. Breast cancer costs in New Zealand's public health system. N Z Med J. 2021;134(1545):36-46.
5. Blakely $\mathrm{T}$ et al. Patterns of cancer care costs in a country with detailed individual data. Med Care. 2015;53(4):302-9. https:// doi.org/10.1097/MLR.0000000000000330

6. Sun L, et al. Global treatment costs of breast cancer by stage: a systematic review. PLoS ONE. 2018. https://doi.org/10.1371/ journal.pone.0207993.

7. Vyas A, et al. Healthcare utilization and costs during the initial phase of care among elderly women with breast cancer. J Natl Compr Cancer Netw. 2017;15(11):1401-9. https://doi.org/10. 6004/jncen.2017.0167.

8. Campbell I, et al. Surgical treatment of early stage breast cancer in the Auckland and Waikato regions of New Zealand. ANZ J Surg. 2018;88(12):1263-8. https://doi.org/10.1111/ans.14840.

9. Caswell-Jin JL, et al. Change in survival in metastatic breast cancer with treatment advances: meta-analysis and systematic review. JNCI Cancer Spectrum. 2018;2(4):pky062. https://doi.org/10. 1093/jncics/pky062.

10. Early Breast Cancer Trialists' Collaborative Group (EBCTCG). Effects of chemotherapy and hormonal therapy for early breast cancer on recurrence and 15-year survival: an overview of the randomised trials. Lancet. 2005;365(9472):1687-717. https://doi. org/10.1016/S0140-6736(05)66544-0.

11. Lawrenson R, et al. The use of trastuzumab in New Zealand women with breast cancer. Asia Pac J Clin Oncol. 2018;14(2):e152-60. https://doi.org/10.1111/j.1445-2197.2011. 05998.x.

12. Seneviratne $\mathrm{S}$, et al. Adherence to adjuvant endocrine therapy: is it a factor for ethnic differences in breast cancer outcomes in New Zealand? Breast. 2015;24(1):62-7. https://doi.org/10.1016/j.clon. 2017.10.015.

13. Lawrenson R, et al. Treatment and survival disparities by ethnicity in New Zealand women with stage I-III breast cancer tumour subtypes. Cancer Causes Control. 2017;28(12):1417-27. https:// doi.org/10.1007/s10552-017-0969-9.

14. Wolff AC, et al. Recommendations for human epidermal growth factor receptor 2 testing in breast cancer: American Society of Clinical Oncology/College of American Pathologists clinical practice guideline update. J Clin Oncol. 2013;31(31):3997-4013. https://doi.org/10.1200/JCO.2013.50.9984.

15. Thuerlimann B. International consensus meeting on the treatment of primary breast cancer 2001, St. Gallen, Switzerland. Breast Cancer. 2001;8(4):294-7. https://doi.org/10.1007/BF02967527.

16. Foster R, et al. Protocol for direct costing of health sector interventions for economic modelling (including event pathways). Wellington: University of Otago; 2012.

17. Ministry of Health. WIESNZ19 cost weights. 2020. https://www. health.govt.nz/nz-health-statistics/data-references/weighted-inlierequivalent-separations/wiesnz19-cost-weights. Accessed 23 Nov 2020.

18. PHARMAC. Pharmaceutical schedule. 2020. https://pharmac. govt.nz/pharmaceutical-schedule/. Accessed 23 Nov 2020.

19. Edge SB, Compton CC. The American Joint Committee on Cancer: the 7th edition of the AJCC cancer staging manual and the future of TNM. Ann Surg Oncol. 2010;17(6):1471-4. https://doi. org/10.1245/s10434-010-0985-4.

20. Brandão M, et al. Healthcare use and costs in early breast cancer: a patient-level data analysis according to stage and breast cancer subtype. ESMO Open. 2020;5(6): e000984. https://doi.org/10. 1136/esmoopen-2020-000984.

21. Blumen H, Fitch K, Polkus V. Comparison of treatment costs for breast cancer, by tumor stage and type of service. Am Health Drug Benefits. 2016;9(1):23-32.

22. Breast Cancer Foundation NZ. Advanced breast cancer. 2021. https://www.breastcancerfoundation.org.nz/breast-cancer/advan ced-breast-cancer. Cited 25 May 2021. 
23. Lao C, et al. Metastatic relapse of stage I-III breast cancer in New Zealand. Cancer Causes Control. 2021. https://doi.org/10.1007/ s10552-021-01426-0.

24. Lawrenson R, et al. Outcomes in different ethnic groups of New Zealand patients with screen-detected vs. non-screen-detected breast cancer. J Med Screen. 2019;26(4):197-203. https://doi. org/10.1177/0969141319844801.

25. Paci E, Duffy S. Overdiagnosis and overtreatment of breast cancer: overdiagnosis and overtreatment in service screening. Breast Cancer Res. 2005;7(6):266-70. https://doi.org/10.1186/bcr1339.

26. Lawrenson $\mathrm{R}$, et al. The use of trastuzumab in New Zealand women with breast cancer. Asia Pac J Clin Oncol. 2017. https:// doi.org/10.1111/ajco.12766.

27. PHARMAC. 12 months sequential treatment of trastuzumab. 2021. https://pharmac.govt.nz/assets/tar-75a-trastuzumab-appen dix-4.pdf. Cited 29 May 2021.
28. Ministry of Health. New cancer registrations 2018. 2020: Wellington, New Zealand.

29. Breast Cancer Foundation NZ. Breast cancer targeted therapy. 2021. https://www.breastcancerfoundation.org.nz/breast-cancer/ treatment-options/targeted-therapy. Cited 25 May 2021.

30. Miller EM, Schwartzberg LS. Biosimilars for breast cancer: a review of HER2-targeted antibodies in the United States. Ther Adv Med Oncol. 2019;11:1758835919887044. https://doi.org/10. 1177/1758835919887044.

31. PHARMAC. Cost resource ManualVersion3-Pharmac. 2018: Wellington, New Zealand. 